\title{
Electrohydrodynamic Motor for Tiny Vessels
}

\author{
Felix M. Moesner, Philipp S. Bühler, Diego C. Politano and Paolo V. Prati \\ Swiss Federal Institute of Technology Zurich (ETHZ) • Institute of Robotics • 8092 Zurich, Switzerland \\ Tel: +41 16323582 •Fax: +411632 1078•Email: moesner@ifr.mavt.ethz.ch
}

Various publications about pumps utilizing electric traveling waves as the conveyor of liquids have been presented [1,2]. In these considerations, a dielectric liquid has been chosen as the media to propel. Inversely, it is conceivable to use pumps as propulsion motors for tiny vessels.

Hereinafter, the electrohydrodynamic propulsion motor is based on the electric tube device which has been introduced in an earlier paper by the first author for the task of particle mass transportation [3]. The device is made by winding 6 parallel and insulated wires to a cylindrical tube. The employed wires have a diameter in the range of $56 \mu \mathrm{m} \sim 236 \mu \mathrm{m}$. Upon the application of 6 phase voltages to the electrodes, the created traveling electric field wave carries the charged liquid in the same direction.

Various electrohydrodynamic motors have been fabricated and optimized through a series of experiments. Optimizing parameters involve electrode-dimensions, fabrication materials, applied voltages and frequencies. As evaluative parameters, the propulsion pressure and the rate of liquid flow is determined. Constant and precise liquid propulsion is achieved. It is further shown that this tube structure has a high potential for miniaturization.

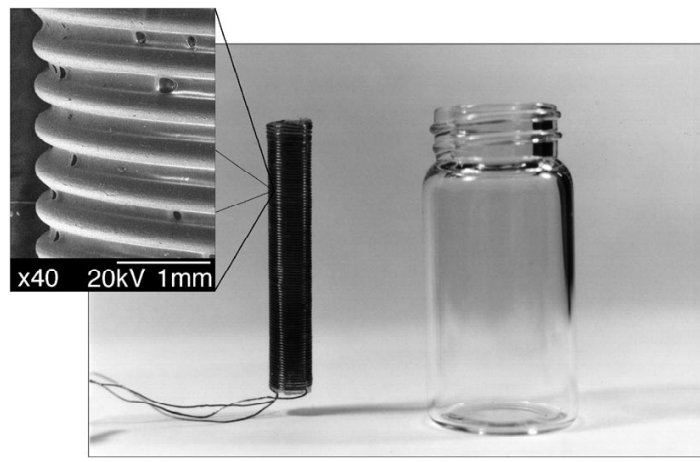

In the conclusion of the presentation, a video will be shown.
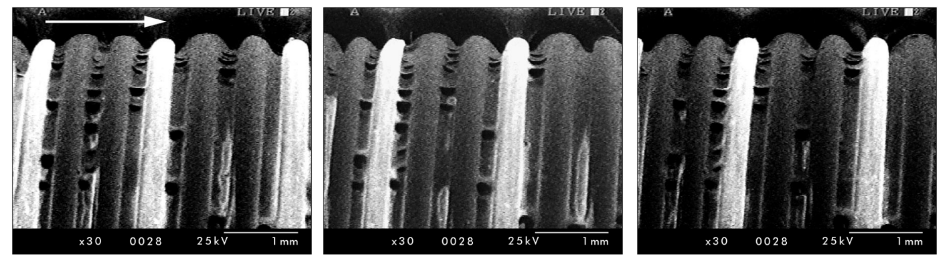

Series of dynamic SEM micrographs showing quantitative negative charge (white) progressing with three-phase traveling wave outside an electrohydrodynamic motor.

Propulsion motor positioned upright next to a specimen container.
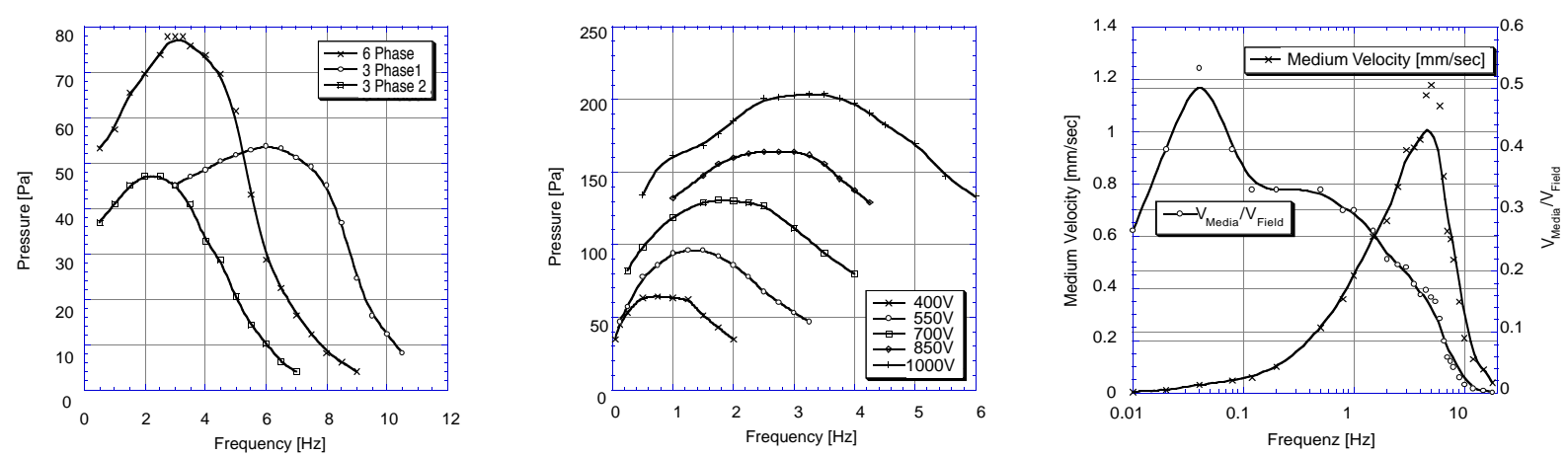

Extract of curves showing propulsion liquid pressure and the rate of liquid flow generation in function of applied frequency at various voltages up to $1 \mathrm{kV}$ amplitude.

[1] Melcher, J.R., "Traveling-Wave Induced Electroconvection," Physics of Fluids, vol. 9, no. 8, 1966, pp. 1548-1555.

[2] Choi, J.W., and Kim, Y.K., "Micro Electrohydrodynamic Pump Driven by Traveling Electric Fields," 30th IAS Annual Meeting Conference Record, 1995, pp. 1480-1484.

[3] Moesner, F.M., and Higuchi, T., "Devices for Particle Handling by an AC Electric Field," Proceedings IEEE Micro Electro Mechanical Systems, Amsterdam, The Netherlands, January 1995, pp. 66-71. 


\title{
Electrohydrodynamic Motor for Tiny Vessels
}

\author{
Felix M. Moesner, Philipp S. Bühler, Diego C. Politano and Paolo V. Prati \\ Swiss Federal Institute of Technology Zurich (ETHZ) • Institute of Robotics \\ 8092 Zurich, Switzerland• Email: moesner@ifr.mavt.ethz.ch
}

\begin{abstract}
Various publications about pumps utilizing electric traveling waves as the conveyor of liquids have been presented. In these considerations, a dielectric liquid has been chosen as the media to propel. Inversely, it is conceivable to use pumps as propulsion motors for tiny vessels.

Hereinafter, the proposed electrohydrodynamic (EHD) motor is based on the electric tube device which has been introduced in an earlier paper by the first author for the task of particle mass transportation [1,2]. The device is made by winding 6 parallel and insulated wires to a cylindrical tube. The employed wires have a diameter in the range of $56 \mu \mathrm{m}$ $236 \mu \mathrm{m}$. Upon the application of multi-phase voltages to the electrodes, the created traveling electric field wave carries the charged liquid in the same direction.

Various EHD propulsion motors have been fabricated and optimized through a series of experiments. Optimizing parameters involve electrode-dimensions, fabrication materials, applied voltages and frequencies. As evaluative parameters, the propulsion pressure and the rate of liquid flow is determined. Constant and precise liquid propulsion is achieved. It is further shown that this tube structure has a high potential for miniaturization.
\end{abstract}

\section{Introduction}

In our modern technological world, miniaturization in many fields has an increasing importance. In this sense, the demand is raising for new kinds of propulsion motors which suit into the microscaled domain. In the current paper, a propulsion motor for a future tiny vessel is presented. The vessel, which will be either a miniature ship or a submarine of maximal a few centimeter length, is intended to incorporate - in a later step - both the autonomous navigation by a microprocessor and the powering of the propulsion. Previously, the problem to find a suitable propulsion motor ready to be mounted to the vessel has to be solved.

In this paper, we focus on the application of electric traveling fields for the propulsion task. Originally, it is not a new idea to use electric fields to move a liquid media. In the sixties, some theoretical models of pumps have been pub-

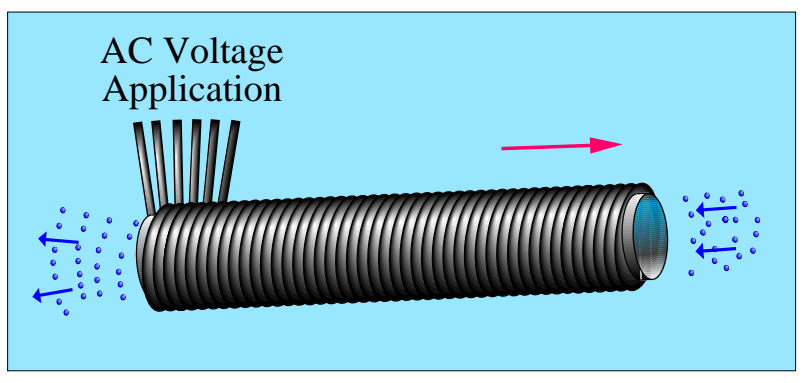

Fig. 1: Schematic of an EHD propulsion motor.

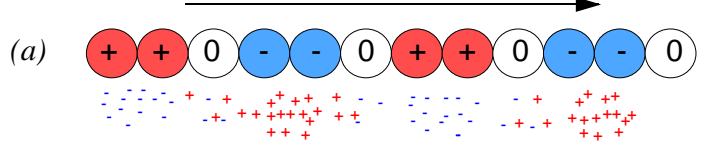

(b)

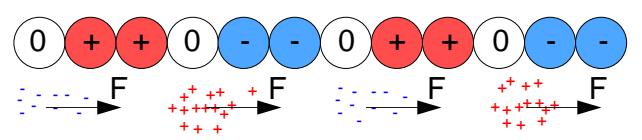

(c)

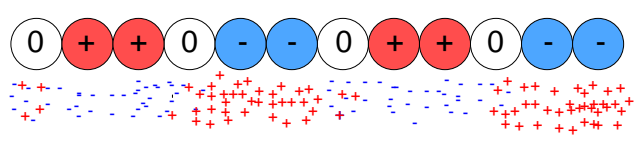

(d)

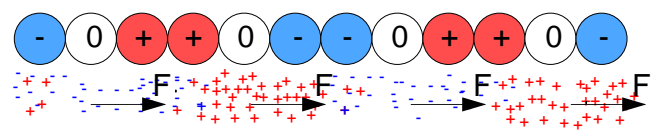

Fig. 2: Principle of the EHD propulsion motor. The liquid around the electrodes becomes charged and is transported in sync with the traveling electric field.
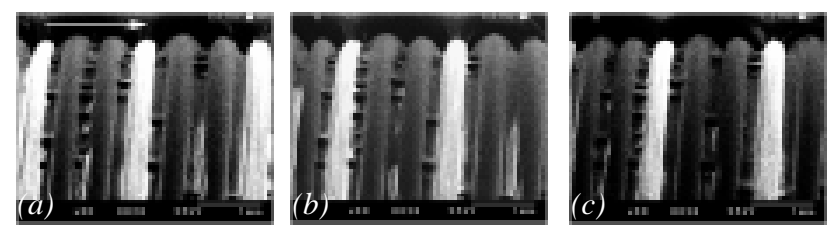

Fig. 3: Series of dynamic SEM micrographs showing quantitative negative charge (white) progressing with three-phase traveling wave outside a propulsion motor. 
lished. Pickard and Stuetzer have proposed a model for an induction-type electrohydrodynamic (EHD) pump [3,4] which has been studied by Melcher in further details $[5,6,7]$. Pumps have also been constructed and tested. In all those cases, large pumps have been designed that function with very high voltages and transport solely insulatorfluids. In recent time, researchers began to built pumps in a smaller scale [8].

The EHD propulsion motor described in this paper is based on the same principle, i.e. inversely, a pump also functions for propulsion purposes. It produces its utmost performance in dielectric liquid revealing some interesting properties. The design itself comprises of structural simplicity. If we consider that the employed method with the traveling electric wave is a surface effect, the electrostatic motor has a very interesting miniaturization potential when compared to a conventional motor following a volume effect. Furthermore, this electrostatic propulsion motor has no moving mechanical parts resulting in no wear and also minimal heat-dissipation - a property indispensable for many applications.

\section{Principle of Electrostatic Propulsion}

The EHD propulsion motor consists of coiled up insulated electrodes connected to a multi-phase voltage source as shown in Figure 1. Upon the application of the phase voltages, an electric field is created around the electrodes. The voltages are supplied in a time-varying manner, producing a traveling electric field.

During operation, the motor is immersed into a dielectric liquid which becomes polarized in the non-uniform electric field and receives a volume charge density corresponding the negative divergence of the polarization. Consequently, the charge is induced into the liquid due to the polarization. The charges interact with the traveling electric field so that the liquid and the charge are moving in direct relation. Figure 2 illustrates the propulsion mechanism. Step a: Polarization of the liquid takes place around the electrodes. Charges get induced into the liquid. Step b: The electric field proceeds to the next electrode pulling the charged liquid in sync. Step c: The liquid becomes repolarized around the electrodes. More and more charges are induced into the liquid. Step d: Again, the electric wave is moving to the next electrode dragging the charged liquid in sync.

An estimation for the appropriate frequency is given by the charge relaxation time $\tau$.

$$
\tau=\varepsilon / \sigma
$$

It is the ratio of the dielectric constant $\varepsilon$ to the electrical
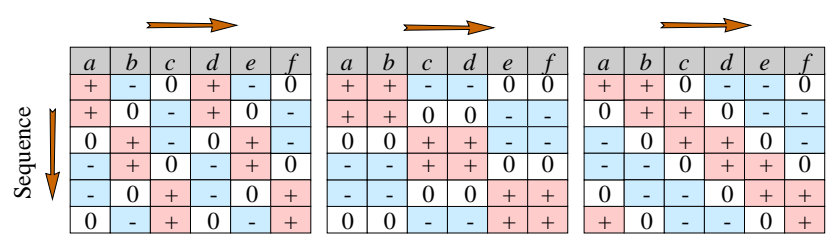

Fig. 4: Three-phase (a), double three-phase (b) and sixphase (c) activation sequences.
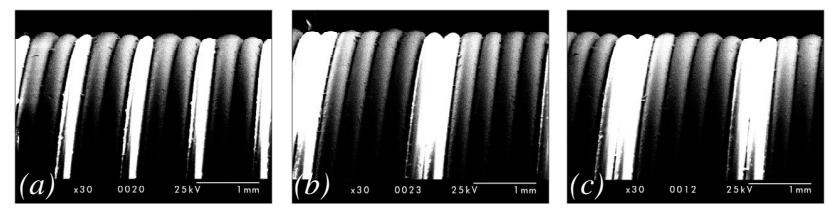

Fig. 5: Charge distribution in three-phase (a), double three-phase (b) and six-phase (c) activation mode.
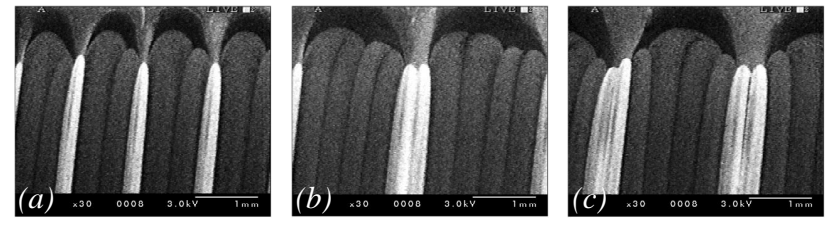

Fig. 6: Same as above. The SEM acceleration voltage is lowered to $3 \mathrm{kV}$, resulting in distorted images.

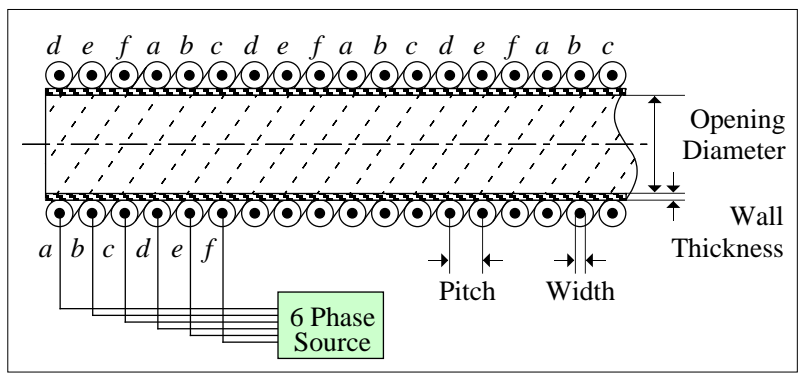

Fig. 7: Cross-section of the EHD propulsion motor illustrating its structural proportions and its six-phase voltage supply attachments

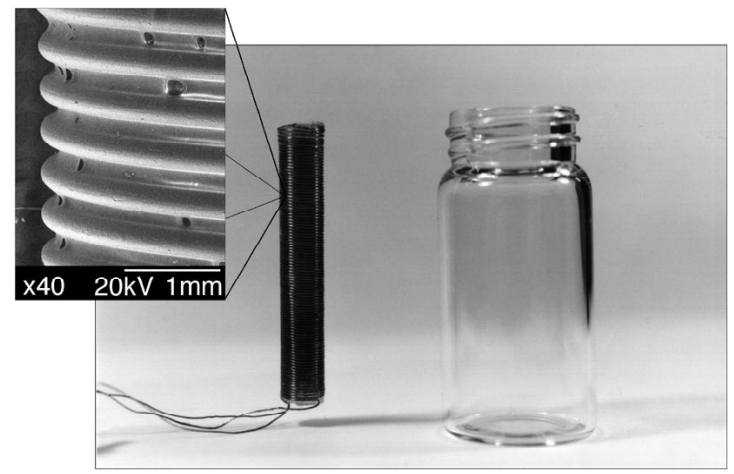

Fig. 8: EHD propulsion motor positioned upright next to a specimen container. 
conductivity $\sigma$ of the liquid. The smaller the ratio, the faster the charge relaxes. The polarization $\boldsymbol{P}$ of a dielectric liquid which is exposed to an electric field $\boldsymbol{E}$ amounts to

$$
\boldsymbol{P}(x, y, z)=\varepsilon \cdot \chi \cdot \boldsymbol{E}(x, y, z)
$$

where $\chi$ is the electric susceptibility of the liquid. The induced charge density at steady state is described by

$$
\rho_{0}=-\nabla \cdot \boldsymbol{P}(x, y, z)
$$

and the instantaneous charge density is expressed by

$$
\rho(x, y, z, t)=\rho_{0} \cdot\left(1-e^{-t / \tau}\right)
$$

where $\tau$ is the charge relaxation time.

The charge $\rho$ is pulled by the advancing traveling electric field $\boldsymbol{E}$ generating a force density $\boldsymbol{F}$ related to the Coulomb force. The produced force is formulated by (5).

$$
\boldsymbol{F}(x, y, z)=\rho \cdot \boldsymbol{E}(x, y, z)
$$

The progression of the traveling electric field can be shown indirectly by a series of scanning electron microscope (SEM) pictures, as presented in Figure 3 for the three-phase voltage application case. The electric wave and its surface charge distribution is traveling stepwise from left (a) to right (c). The white area represents negative charge. Among the applied multi-phase voltages, the three-phase, the double three-phase and the six-phase activation sequences, which are shown in Figure 4, have been chosen. The corresponding SEM images are presented in Figure 5 and 6 . The latter pictures help to distinguish between the actual applied voltages to the single electrodes.

\section{Fabrication}

The main advantage of the tube shape structure is the simple fabrication of the EHD propulsion motor. Polyimide insulated copper wires are spirally wound up onto a cylindrical substrate, fixed by a bond and finally removed from the substrate. Table 1 contains all motors with information on electrode width / pitch and type of fixation bond.

Tab. 1: Employed bonding materials and wire diameters

\begin{tabular}{|l|c|c|c|c|}
\hline $\begin{array}{c}\text { Electrode Width/ } \\
\text { Bond Pitch }\end{array}$ & $\begin{array}{c}56 / 63 \\
\mu \mathrm{m}\end{array}$ & $\begin{array}{c}132 / 145 \\
\mu \mathrm{m}\end{array}$ & $\begin{array}{c}190 / 200 \\
\mu \mathrm{m}\end{array}$ & $\begin{array}{c}236 / 250 \\
\mu \mathrm{m}\end{array}$ \\
\hline \hline Cyanoacrylate & $\checkmark$ & $\checkmark$ & $\checkmark$ & $\checkmark$ \\
\hline Epoxy & & $\checkmark$ & $\checkmark$ & $\checkmark$ \\
\hline Adhesive Tape & & & & $\checkmark$ \\
\hline Urethane Spray & & & $\checkmark$ & $\checkmark$ \\
\hline
\end{tabular}

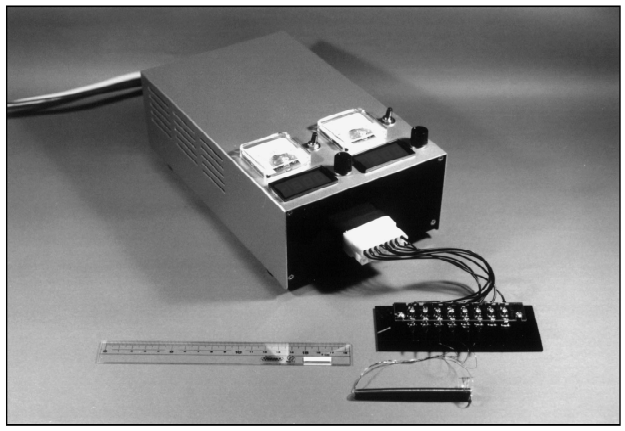

Fig. 9: The EHD propulsion motor is attached to the PC controlled voltage source via a connection bridge.

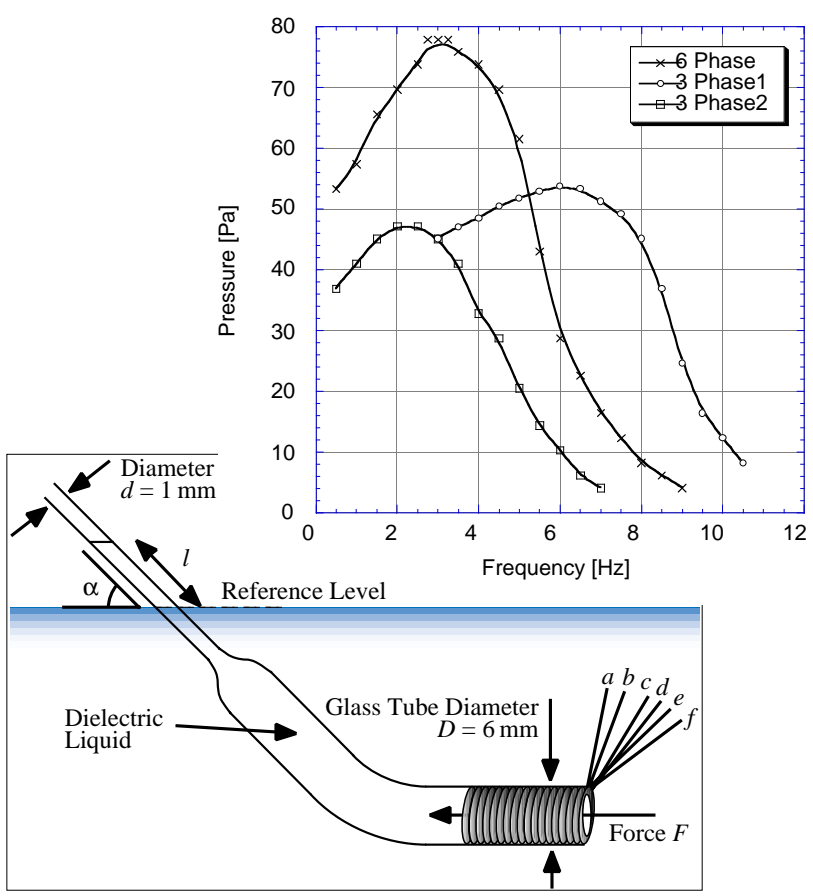

Fig. 10: Propulsion liquid pressure vs applied frequency for the multi-phase case. Schematic of setup used for measuring the propulsion pressure.

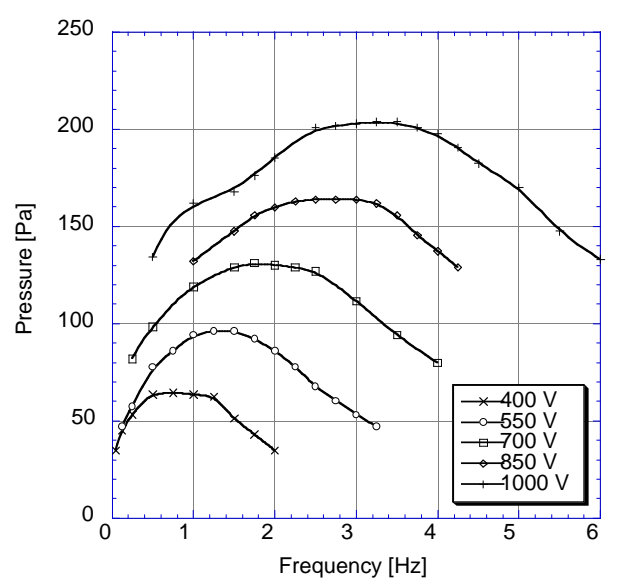

Fig. 11: Propulsion liquid pressure vs applied frequency for a set of voltage amplitudes. 
Figure 7 shows the cross-section of the EHD propulsion motor. Among the fixation bonds, the adhesive tape proofed to be impracticable through its self-dissolving character when immersed into the liquid. The fixation with urethane spray produced a structure with insufficient stiffness. The usage of both the cyanoacrylate and the epoxy compound resulted in successful devices whose sample is depicted in Figure 8. The complete system including the voltage source is shown in Figure 9.

\section{Propulsion Pressure}

In a first experiment to evaluate the performance of the EHD propulsion motor, the liquid propulsion pressure is determined. The motor is inserted into a curved glass tube as shown in Figure 10 and immersed into the liquid (corn oil). The internal space of the motor is filled with a concentric solid cylinder leaving enough space for the liquid to pass. The produced pressure $p$ is calculated as

$$
p=\sin \alpha \cdot l \cdot \rho_{\text {liquid }} \cdot g
$$

where $\alpha$ is the incline angle of the glass tube, $l$ is the climbing distance of the liquid, $\rho_{\text {liquid }}$ is the density of the liquid and $g$ is the gravitational acceleration. The measurement results are presented in the graphs of Figure 10 and 11. As expected, the six-phase voltage application produces the greatest effectiveness. Furthermore it is stated, that the generated pressure is in clear relation to the applied voltage amplitude and to the frequency.

\section{Rate of Liquid Flow}

The liquid flow rate of the motor is measured in a glass tube. A marker helps to evaluate the liquid velocity $v_{\text {Media }}$ as illustrated in Figure 12. The flow rate $Q$ is

$$
Q=v_{\text {Media }} \cdot(D / 2)^{2} \cdot \pi \text {. }
$$

The six-phase traveling wave velocity is calculated by

$$
v_{\text {Field }}=6 \cdot \text { pitch } \cdot f \text {. }
$$

The experimental results for a $250 \mu \mathrm{m}$ pitched motor are presented in Figure 13.

\section{Conclusion}

The best performance have been achieved for a $190 \mu \mathrm{m}$ pitched, epoxy designed motor. A max. pressure of $205 \mathrm{~Pa}$ is reached with six-phase voltage application at $1 \mathrm{kV}$ amplitude and at $3.5 \mathrm{~Hz}$. The max. flow rate is $31.5 \mu \mathrm{l} / \mathrm{sec}$ at $0.8 \mathrm{kV}$ amplitude and at $3 \mathrm{~Hz}$. Substantial performance improvement can be attained by a concatenation of concentric EHD propulsion motors.

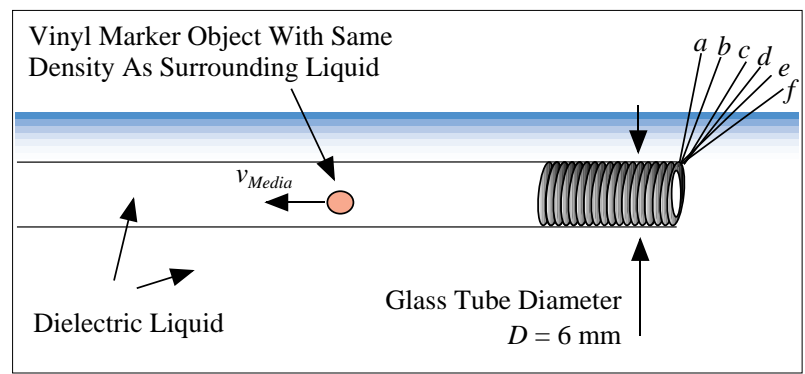

Fig. 12: Schematic of setup used for measuring the rate of liquid flow.

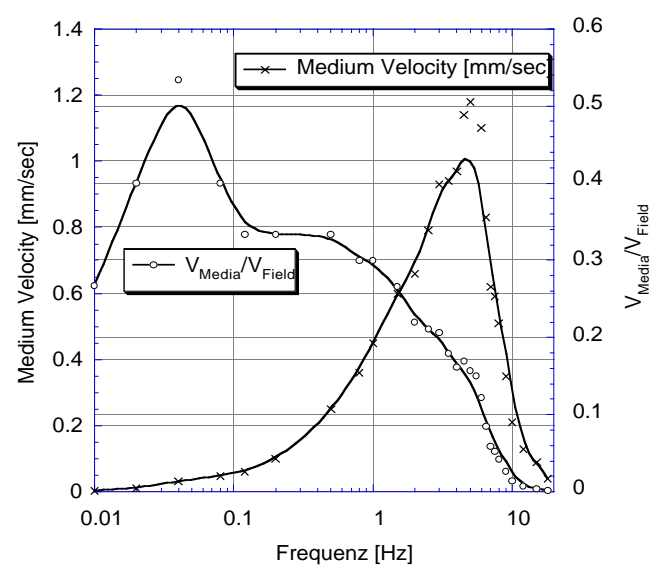

Fig. 13: Rates of liquid flow and ratio of flow rate to wave propagation velocity vs applied frequency. An amplitude of $1 \mathrm{kV}$ is applied to $250 \mu \mathrm{m}$ pitched electrodes.

\section{References}

[1] Moesner, F.M., and Higuchi, T., "Devices for Particle Handling by an AC Electric Field," Proceedings IEEE Micro Electro Mechanical Systems, Amsterdam, The Netherlands, January 1995, pp. 66-71.

[2] Moesner, F.M., "Transportation and Manipulation of Particles by an AC Electric Field," Doctoral Thesis ETH No.11961, Swiss Federal Institute of Technology, Zurich, 1996.

[3] Pickard, W.F., "Ion-Drag Pumping In Theory," Journal of Applied Physics, Vol. 34, 1963, pp. 136-146.

[4] Stuetzer, O.M., "Ion-Drag Pumps," Journal of Applied Physics, Vol. 34, 1963, pp. 136-146.

[5] Melcher, J.R., "Traveling-Wave Induced Electroconvection," Physics of Fluids, Vol. 9, No. 8, 1966, pp. 1548-1555.

[6] Melcher, J.R., "Traveling-Wave Bulk Electroconvection Induced across a Temperature Gradient," Physics of Fluid, Vol.10, No6,1967, pp. 1178-1185.

[7] Melcher, J.R., "Electric Fields and Forces in Semi-Insulating Liquids," Journal of Applied Physics, Vol. 2, 1976, pp. 121-132.

[8] Choi, J.W., and Kim, Y.K., "Micro Electrohydrodynamic Pump Driven by Traveling Electric Fields," 30th IAS Annual Meeting Conference Record, 1995, pp. 1480-1484. 